\title{
VARIACIÓN ANATÓMICA Y MORFOLÓGICA EN ESPECIES Y ENTRE POBLACIONES DE OPUNTIA EN LA PORCIÓN Sur del Desierto ChiHuahuense
}

\author{
Alejandro Muñoz-Urias ${ }^{1,5}$, Guadalupe Palomino-Hasbach ${ }^{2}$, Teresa Terrazas ${ }^{3}$, \\ Armando García-VelázQuez ${ }^{4}$ y Eulogio Pimienta-Barrios ${ }^{1}$
}

'Departamento de Ecología, Universidad de Guadalajara, km 15.5 carretera Guadalajara Nogales, Zapopan, Jalisco, México

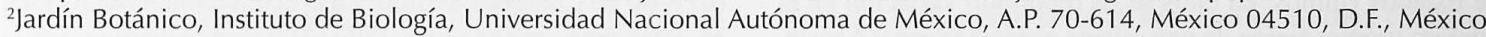
${ }^{3}$ Departamento de Botánica, Instituto de Biología, Universidad Nacional Autónoma de México, A.P. 70-633,

México 04510 D.F., México

${ }^{4}$ Instituto de Recursos Genéticos y Productividad, Colegio de Postgraduados,

km 36.5 Carretera México-Texcoco, Montecillo 56230, México

${ }^{5}$ Autor para la correspondencia. Correo-e: almunoz@cucba.udg.mx

\begin{abstract}
Resumen: Se evaluó la variación fenotípica a través de 28 variables anatómicas y morfológicas mediante análisis discriminantes en poblaciones de Opuntia cantabrigiensis, $O$. leucotrich,$O$. rastrera, $O$. robusta y $O$. streptacantha que se encuentran separadas entre sí por distancias de entre 32 y $110 \mathrm{~km}$. La altitud y la temperatura media anual no mostraron variación en el área de estudio. El análisis separa las poblaciones de $O$. cantabrigiensis, que es la especie con menor densidad (13 individuos ha ${ }^{-1}$ ), mientras que para el resto de las especies, cuyas poblaciones pueden alcanzar hasta 1,852 individuos ha $^{-1}$, algunas poblaciones se yuxtaponen. No hay diferencias en los niveles de ploidía entre las poblaciones, a pesar de que se registraron niveles de ploidía entre diploide (O. cantabrigiensis) a octoploide $(O$. streptacantha). Por lo tanto, las diferencias fenotípicas entre las poblaciones parecen estar relacionadas con la densidad de individuos y no con variables ambientales, dada la falta de gradientes ambientales o a cambios en el nivel de ploidía de las distintas especies.
\end{abstract}

Palabras clave: ambiente semiárido, números cromosómicos, Opuntia, variación morfológica, variación anatómica.

Abstract: Phenotypic variation in different populations of Opuntia cantabrigiensis, O. leucotricha, O. rastrera, O. robusta and $O$. streptacantha was evaluated by examining 28 anatomical and morphologic variables through discriminant analysis. These populations are separated from each other by distances ranging between 32 and $110 \mathrm{~km}$. Altitude and annual average temperature showed no variation in the study area. Discriminant analysis separated populations of $O$. cantabrigiensis, which is the species having the lowest density ( 13 individuals ha ${ }^{-1}$ ), whilst some populations of other Opuntia species, whose densities were as high as 1,852 individuals ha $^{-1}$, overlapped. No different ploidy levels were found among populations, although we recorded levels of ploidy between species that ranged from diploid (O. rastrera) to octoploid $(O$. streptacantha). Therefore, phenotypic differences between populations appear to be related to the density of individuals rather than to environmental variables, given the lack of environmental gradients or change in level ploidy.

Key words: Anatomical and morphological variation, chromosome number, Opuntia, semi-arid environments.

$E^{\prime}$ género Opuntia se distribuye principalmente en regiones semiáridas, bosques mésicos, pastizales y bosques tropicales (Starmer et al., 2003). Sin embargo, es en zonas áridas y semiáridas donde se observa la mayor riqueza de especies de este género. En México, el Altiplano PotosinoZacatecano que comprende partes territoriales de los estados de Aguascalientes, Guanajuato, Querétaro, Jalisco y Duran- go, se caracteriza por la presencia de extensas áreas en las que la comunidad vegetal está constituida por miembros de este género (Marroquín et al., 1964). En estas áreas, Opuntia muestra una marcada variación morfológica al grado de que su taxonomía se vuelve confusa (Bravo-Hollis, 1978; Scheinvar, 1995). Es común que haya variación en los hábitos de crecimiento, tiempos de presentación de las fenofases 
vegetativas y reproductivas, así como en la forma, el color, el peso y la composición química del fruto (Wallace y Fairbrothers, 1986; Pimienta-Barrios y Mauricio-Leguizamo, 1989; Pimienta-Barrios, 1994; Pimienta-Barrios y MuñozUrias, 1995) y en los niveles de ploidía (Muñoz-Urias et al., 1995). No obstante, el estudio de las causas de esta variación ha recibido poca atención (Gibson y Nobel, 1986). La variación presente en especies silvestres y cultivadas de este género ha sido relacionada con fenómenos como la hibridación, asociada a un incremento de poliploidía, y con el aislamiento geográfico, por lo cual es importante estudiar estos fenómenos para el mejor entendimiento de la variabilidad del género (Gibson y Nobel, 1986; Pimienta-Barrios y Muñoz-Urias, 1995; Scheinvar, 1995).

En varios estudios se han registrado niveles de ploidía de las opuntias que crecen en el norte de México y el sur de Estados Unidos; en ellos se ha reportado variación en el número cromosómico de una misma especie (Sosa y Acosta, 1966; Pinkava y Mc Leod, 1971; Pinkava et al., 1973; Mc Leod, 1975; Pinkava et al., 1977, 1985, 1992; Palomino y Heras, 2001). Con respecto a la hibridación, se han reportado numerosos híbridos en este género (Mc Leod, 1975; Grant y Grant, 1979; 1980; Parffit, 1980; Pinkava et al., 1992; Mayer et al., 2000; Griffith, 2001). Sin embargo, el efecto del aislamiento geográfico no ha sido evaluado, no obstante que éste se ha incrementado, debido a que en los últimos años se ha observado una disminución de las nopaleras debido a la fragmentación de las poblaciones silvestres causada por la apertura de nuevas tierras para las actividades agrícolas. Este aislamiento puede tener como consecuencia un incremento de la erosión genética, la reducción del flujo genético entre poblaciones y la deriva génica, promoviendo diferenciación entre poblaciones (Gastó et al., 1981; Janzen, 1986; Ellstrand y Elam, 1993; Young y Clarke, 2000).

En este estudio se determinaron los números cromosómicos y se evaluaron variables morfológicas y anatómicas, así como las densidades poblacionales, en cinco de las especies silvestres de Opuntia más importantes que forman parte del paisaje de las zonas semiáridas del Altiplano Potosino-Zacatecano: O. cantabrigiensis Lynch, O. leucotricha DC., $O$. streptacantha Lem., O. rastrera Weber y O. robusta $\mathrm{H}$. L.Wendl. Partimos de la hipótesis de que si la variación morfológica presente en las especies de Opuntia es generada por la hibridación natural, es de esperarse que los fenotipos de los híbridos de las especies de Opuntia en estudio muestren características morfológicas intermedias con los progenitores. Por lo tanto, se observará yuxtaposición entre las especies cuando se lleve a cabo el análisis discriminante entre éstas. Además, se evaluó la variación entre poblaciones aisladas, con la finalidad de observar la plasticidad fenotípica, y se determinó si existen citotipos cromosómicos entre las poblaciones que se vean reflejados en cambios en la morfología de las plantas. Se pretendió responder las siguientes preguntas: (1) ¿existe hibridación natural entre individuos de diferentes especies?, (2) ¿hay diferencias fenotípicas en poblaciones de Opuntia aisladas por varios kilómetros?, (3) si existe divergencia en la morfología, ¿será más notable en poblaciones de menor densidad a causa de la deriva génica?, (4) si existen poblaciones dentro de una misma especie con diferente número cromosómico ¿habrá diferencias morfológicas entre plantas de la misma especie pero con diferente número cromosómico?

\section{Materiales y métodos}

Descripción del área de estudio. Este estudio se llevó a cabo en la región de los Llanos de Ojuelos, en la altiplanicie semiárida de la parte suroeste del Desierto Chihuahuense. Las áreas de estudio fueron (a) El Rayo, Zacatecas; (b) Laguna de Guadalupe, Guanajuato; (c) Villa Hidalgo, Zacatecas; y (d) Paso de Cuarenta, Jalisco. La precipitación pluvial anual en estas áreas oscila entre 450 y $500 \mathrm{~mm}$, mientras que la temperatura anual promedio es de 16 a $18^{\circ} \mathrm{C}$. El clima de las cuatro localidades puede ser clasificado como seco estepario templado en el sistema de Köppen (Pimienta-Barrios, 1990). En el cuadro 1 se sintetizan los datos de la ubicación geográfica y dos variables del clima, y en el cuadro 2 se muestran las distancias entre las áreas de estudio.

Descripción del material vegetativo. Las especies que se estudiaron (Opuntia cantabrigiensis, O. leucotricha, O. rastrera, $O$. robusta y $O$. streptacantha) son de amplia distribución en la porción sur del Desierto Chihuahuense. Crecen comúnmente de forma simpátrica o parapátrica y presentan una amplia variabilidad morfológica (Britton y Rose 1963; Bravo-Hollis, 1978). (a) O. cantabrigiensis es un arbusto redondeado, de 1 a $2 \mathrm{~m}$ de altura, con cladodios de forma orbicular hasta obovada, de 12 a $20 \mathrm{~cm}$ de longitud, de color verde azulado. (b) O. leucotricha es una planta arborescente de 3 a $5 \mathrm{~m}$ de altura, con tronco más o menos bien definido y ramoso, cubierto con pelos setosos, blancos y flexibles; sus cladodios son de forma oblonga, de 15 a $30 \mathrm{~cm}$ de largo y de epidermis pubescente. (c) $O$. rastrera es una planta rastrera con artículos circulares hasta obovados, los más grandes de unos $20 \mathrm{~cm}$ de diámetro, que forma grandes cadenas. (d) $O$.

Cuadro 1. Características climáticas de las localidades de estudio.

\begin{tabular}{lcccc}
\hline Localidad & $\begin{array}{c}\text { Altitud } \\
(\mathrm{m} \text { s.n.m. })\end{array}$ & $\begin{array}{c}\text { Latitud, } \\
\text { longitud }\end{array}$ & $\begin{array}{c}\text { Precipitación } \\
\text { pluvial (mm) }\end{array}$ & $\begin{array}{c}\text { Temperatura } \\
\text { media anual } \\
\left({ }^{\circ} \mathrm{C}\right)\end{array}$ \\
\hline $\begin{array}{l}\text { Paso de } \\
\text { Cuarenta }\end{array}$ & 1,930 & $\begin{array}{l}21^{\circ} 31^{\prime} \mathrm{N}, \\
10^{\circ} 41^{\prime} \mathrm{O}\end{array}$ & 535.2 & 17.45 \\
Villa Hidalgo & 2,150 & $\begin{array}{l}22^{\circ} 25^{\prime} \mathrm{N}, \\
101^{\circ} 25^{\prime} \mathrm{O}\end{array}$ & 427.8 & 16.25 \\
& & & \\
Laguna de & 2,180 & $\begin{array}{l}21^{\circ} 49^{\prime} \mathrm{N}, \\
101^{\circ} 22^{\prime} \mathrm{O}\end{array}$ & 433.8 & 16.11 \\
$\begin{array}{l}\text { Guadalupe } \\
\text { El Rayo }\end{array}$ & 2,190 & $\begin{array}{l}21^{\circ} 58^{\prime} \mathrm{N}, \\
101^{\circ} 35^{\prime} \mathrm{O}\end{array}$ & 542.2 & 16.65 \\
\hline
\end{tabular}


Cuadro 2. Distancias entre poblaciones evaluadas dentro del área de estudio.

\begin{tabular}{lccc}
\hline & $\begin{array}{c}\text { Villa de Hidalgo } \\
(\mathrm{km})\end{array}$ & $\begin{array}{c}\text { Laguna de Guadalupe } \\
(\mathrm{km})\end{array}$ & $\begin{array}{c}\text { El Rayo } \\
(\mathrm{km})\end{array}$ \\
\hline Paso de Cuarenta & 110 & 47 & 50 \\
Villa de Hidalgo & & 75.5 & 58 \\
Laguna de Guadalupe & & & 32 \\
\hline
\end{tabular}

robusta es arbustiva, muy ramificada, de 1 a $2 \mathrm{~m}$ de altura; su tronco está más o menos definido, con ramas como de $1.5 \mathrm{~m}$ de largo y cladodios orbiculares o algo oblongos, de 15 a $40 \mathrm{~cm}$ de longitud o más. (e) O. streptacantha es arborescente, muy ramificada, hasta de $5 \mathrm{~m}$ de altura, con tronco bien definido, a veces hasta $45 \mathrm{~cm}$ de diámetro; sus cladodios tienen formas obovados hasta orbiculares, de 25 a 30 cm de longitud (Britton y Rose, 1963; Bravo-Hollis, 1978). Evaluación morfológica y anatómica. Los rasgos morfológicos y anatómicos de cladodios maduros (cuadro 3, figura 1) fueron evaluados en plantas separadas entre sí por una distancia de $10 \mathrm{~m}$. Los muestreos se realizaron en 20 plantas por especie, excepto en Laguna y Villa Hidalgo de Guada-

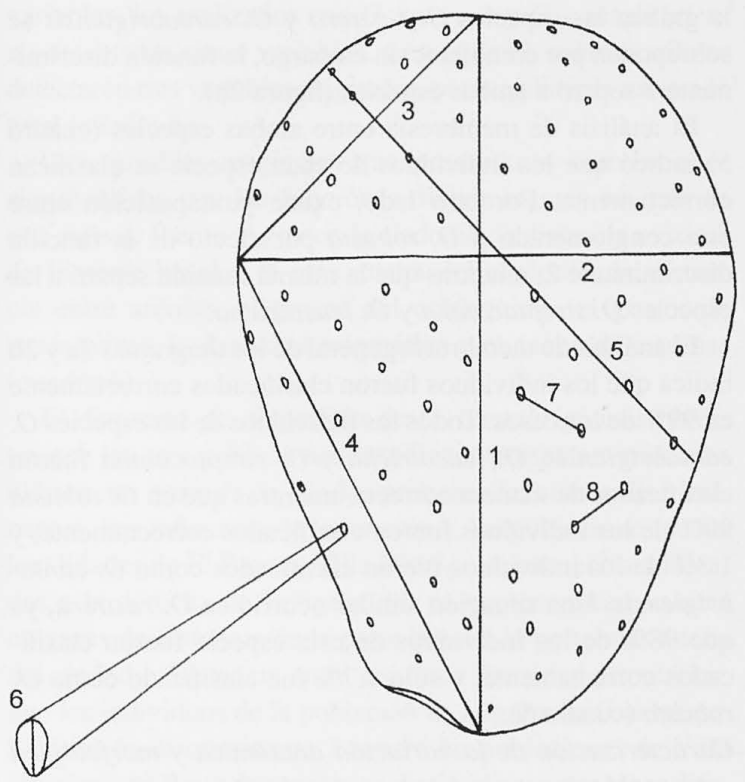

Figura 1. Caracteres morfológicos analizados en cladodios de Opuntia. (1) largo del cladodio (cm); (2) ancho del cladodio (cm); (3) distancia máxima de la parte apical a la más ancha $(\mathrm{cm}) ;(4)$ distancia máxima de la parte basal a la mas ancha $(\mathrm{cm})$; (5) número de líneas de aréolas; (6) tamaño de la aréola; (7) distancia entre aréolas $(\mathrm{cm}) ;(8)$ distancia entre líneas $(\mathrm{cm})$. lupe, ya que allí se recolectaron alrededor de 10 individuos de $O$. cantabrigiensis y $O$. leucotricha debido a la escasez de material; además, en Paso de Cuarenta no se recolectaron muestras de $O$. cantabrigiensis, $O$. leucotricha y $O$. rastrera debido a la ausencia de estas especies en esta localidad. Sólo se registró un cladodio que estuviera en floración o fructificación, y que no presentaran daños causados por factores bióticos y abióticos. Además se recolectaron y herborizaron tres ejemplares por especie, los cuales fueron depositados en el Herbario del Instituto de Botánica de la Universidad de Guadalajara (IBUG).

Para el estudio anatómico del córtex, se disectaron tiras epidermales de aproximadamente $1 \mathrm{~cm}$ de longitud de la parte central del cladodio. Éstas fueron deshidratadas en alcohol $(96 \%)$ por $24 \mathrm{~h}$. Una vez deshidratadas, se tiñeron con azul de toluidina $(0.05 \% \mathrm{v} / \mathrm{v})$ por 5 a $10 \mathrm{~min}$. Además, se hicieron cortes transversales en diez cladodios de cada especie en cada población, para lo cual se empleó un sacabocados de $7.8 \mathrm{~mm}$ de diámetro. En estas muestras se hicieron cortes trasversales de aproximadamente $80 \mu \mathrm{m}$ con una navaja de rasurar. Éstos fueron teñidos con azul de toluidina $(0.5 \% \mathrm{v} / \mathrm{v})$ de 5 a $10 \mathrm{~min}$. En cada corte se registró información de dos campos oculares y para medir el tamaño $(\mu \mathrm{m})$ se empleó un microscopio óptico (Zeiss) al que se le adaptó un micrómetro ocular. Los rasgos morfológicos y anatómicos evaluados se seleccionaron con base en los trabajos de Orea-Rosas (1986) y Muñoz-Urias et al. (1995) y se enlistan en el cuadro 3.

Densidad de las poblaciones y determinación de los números cromosómicos. Se determinó la densidad en las cuatro localidades, para lo cual se utilizaron tres parcelas rectangulares de $18 \times 30 \mathrm{~m}\left(540 \mathrm{~m}^{2}\right)$ en los que se registraron todos los individuos de cada una de las especies (Brower et al., 1997). Para la determinación de los números cromosómicos se recolectaron ápices de raíces en crecimiento de tres plantas por especie y por población, y se colocaron en 8-hidroxiquinoleína fría $(0.002 \mathrm{M})$ por $6 \mathrm{~h}$. A continuación, los ápices radicales fueron hidrolizados con ácido clorhídrico $(1 \mathrm{~N})$ por $10 \mathrm{~min}$ a $60^{\circ} \mathrm{C}$ y se trasfirieron posteriormente al reactivo de Feulgen por $7 \mathrm{~min}$ a $60^{\circ} \mathrm{C}$. Se elaboraron preparaciones por medio de la técnica de squash para observar cinco células en metafase (García Velázquez, 1988).

Análisis estadístico. Antes de llevar a cabo el análisis discriminante se probó que las variables presentaran distribución normal por medio de la prueba estadística de Kolmogorov-Smirnov (Zar, 1984). En los casos en los que no se presentó distribución normal los datos fueron transformados con logaritmo natural, $\mathrm{x}^{2}$ o raíz cuadrada. Las variables largo y ancho de cladodio, número de espinas entre 1 y 3 $\mathrm{cm}$, número de espinas mayores de $3 \mathrm{~cm}$, número de espinas rectas por aréola y número de espinas reflejas por aréola no 
presentaron distribución normal después de las transformaciones, por lo cual fueron eliminadas.

Se llevó a cabo el análisis discriminante múltiple utilizando el programa SPSS versión 10 para determinar si existen diferencias entre poblaciones aisladas y entre especies a través de variables anatómicas y morfológicas; este análisis se utiliza para asignar casos a un grupo definido por una anterior clasificación y recientemente, para evaluar una clasificación sobre la base de un cierto número de atributos. Esta técnica busca funciones discriminantes que maximizan las diferencias entre los grupos y se ha utilizado para determinar posible hibridación (Valverde et al., 1996; Vite et al., 1996; Zelaya, 2007). Además, se llevó a cabo un análisis de membresía, el cual predice la entidad de cada individuo con base en sus puntuaciones discriminantes y las compara con las entidades reales en este caso especies y poblaciones, como medida de efectividad del análisis (Hair et al., 1999).

\section{Resultados}

Caracterización de la variación anatómica y morfológica entre especies silvestres de Opuntia. El análisis discriminante explicó $94 \%$ de la variación total en las tres primeras funciones discriminantes. El valor propio de la primera función discriminante explicó $60 \%$ de la variación; las variables originales correlacionadas con esta función fueron la pro-

Cuadro 3. Variables morfológicas y anatómicas observadas en cladodios maduros en Opuntia spp.

\begin{tabular}{|c|c|}
\hline \multicolumn{2}{|l|}{ Variables morfológicas y anatómicas } \\
\hline $\begin{array}{l}\text { (1) largo del cladodio (cm) (Fig. } \\
\text { 1) } \\
\text { (2) ancho del cladodio (cm) (Fig. } \\
\text { 1) } \\
\text { (3) distancia máxima de la parte } \\
\text { apical a la más ancha (cm) (Fig. } \\
\text { 1) } \\
\text { (4) distancia máxima de la parte } \\
\text { basal a la más ancha (cm) (Fig. 1) } \\
\text { (5) número de líneas de aréolas } \\
\text { por cara del cladodio (Fig. 1) } \\
\text { (6) tamaño de la aréola (mm) } \\
\text { (Fig. } 1 \text { ) } \\
\text { (7) distancia entre aréolas (cm) } \\
\text { (Fig. } 1 \text { ) } \\
\text { (8) distancia entre líneas (cm) } \\
\text { (Fig. } 1 \text { ) } \\
\text { (9) grosor del cladodio (cm) } \\
\text { (10) altura del tallo (cm) } \\
\text { (11) tamaño del gloquidio } \\
\text { (12) número de espinas menores } \\
\text { de } 1 \mathrm{~cm} \\
\text { (13) número de espinas entre } 1 \\
\text { y } 3 \mathrm{~cm} \\
\text { (14) número de espinas mayores } \\
\text { a } 3 \mathrm{~cm} \\
\text { (15) número total de espinas por } \\
\text { aréola. }\end{array}$ & $\begin{array}{l}\text { (16) número de espinas rectas } \\
\text { por aréola (espinas que forman } \\
\text { un ángulo de } 80 \text { a } 90^{\circ} \text { con la } \\
\text { superficie del cladodio) } \\
\text { (17) número de espinas porrectas } \\
\text { por aréola (espinas que forman } \\
\text { un ángulo de } 46 \text { a } 75^{\circ} \text { con la } \\
\text { superficie del cladodio) } \\
\text { (18) número de espinas reflejas } \\
\text { (espinas que forman un ángulo } \\
\text { de } 16 \text { a } 40^{\circ} \text { con la superficie del } \\
\text { cladodio) } \\
\text { (19) número de espinas adpresas } \\
\text { (espinas que forman un ángulo de } \\
\text { 10 o menos con la superficie del } \\
\text { cladodio) } \\
\text { (20) grosor de la cutícula }(\mu \mathrm{m}) \\
(21) \text { grosor de la epidermis }(\mu \mathrm{m}) \\
\text { (22) grosor del colénquima }(\mu \mathrm{m}) \\
(23) \text { grosor del parénquima }(\mu \mathrm{m}) \\
(24) \text { profundidad de la cripta } \\
\text { estomática ( } \mu \mathrm{m}) \\
(25) \text { densidad estomática } \\
\text { (estomas·mm) } \\
(26) \text { largo de estomas }(\mu \mathrm{m}) \\
(27) \text { ancho de estomas }(\mu \mathrm{m}) \\
(28) \text { área de estomas }\left(\mu \mathrm{m}^{2}\right)\end{array}$ \\
\hline
\end{tabular}

fundidad de la cripta estomática, el grosor de cutícula, la altura del tallo y el número de espinas adpresas por aréola. El valor propio para la segunda función representó $23 \%$ de la variación. Esta función discriminante estuvo relacionada con el número de espinas por aréola menores de $1 \mathrm{~cm}$, el grosor del parénquima, el grosor del cladodio y el grosor de la epidermis, mientras que la tercer función explicó $6.4 \%$ de la variación y se correlacionó con el número de líneas de aréolas por cara del cladodio, la distancia máxima de la parte apical a la más ancha del cladodio, la distancia entre líneas de aréolas, la distancia máxima de la parte basal a la más ancha del cladodio, el ancho de estomas y el grosor del colénquima (cuadro 4).

El diagrama de dispersión muestra que las funciones discriminantes 1 y 2 explicaron en conjunto $88 \%$ de la variación (figura 2a). La función discriminante 1 distribuyó hacia el lado derecho de la gráfica a $O$. leucotricha y $O$. streptacantha, separándolas del resto de las especies, ya que éstas poseen mayor profundidad de la cripta estomática, grosor de cutícula, altura del tallo y número de espinas adpresas por aréola. La función discriminante 2 separó verticalmente a $O$. streptacantha de $O$. leucotricha, ya que la primera de estas dos especies se caracteriza por presentar mayor grosor de parénquima, del cladodio y de la epidermis. De igual forma, tiene menor número de espinas menores de $1 \mathrm{~cm}$ por aréola. Así mismo, se observa que en el lado izquierdo de la gráfica las especies $O$. rastrera y $O$. cantabrigiensis se sobreponen por completo; sin embargo, la función discriminante 3 separó a ambas especies (figura $2 b$ ).

El análisis de membresía entre ambas especies (cuadro 5) indicó que los individuos de cada especie se clasifican correctamente. Por otro lado, existe yuxtaposición entre este conglomerado y $O$. robusta por efecto de la función discriminante 2 , mientras que la misma función separó a las especies $O$. streptacantha y $O$. leucotricha.

El análisis de membresía general de los diagramas $2 a$ y $2 b$ indica que los individuos fueron clasificados correctamente en $99 \%$ de los casos. Todos los individuos de las especies $O$. cantabrigiensis, O. leucotricha y O. streptacantha fueron clasificados de manera correcta, mientras que en $O$. robusta 99\% de los individuos fueron clasificados correctamente, y $1.3 \%$ de los individuos fueron clasificados como $O$. cantabrigiensis. Una situación similar ocurrió en $O$. rastrera, ya que $98 \%$ de los individuos de esta especie fueron clasificados correctamente, y sólo $1.7 \%$ fue clasificado como $O$. robusta (cuadro 5).

Caracterización de la variación anatómica y morfológica entre poblaciones de las especies de Opuntia. Los análisis discriminantes sobre la caracterización de la variación anatómica y morfológica de las especies de Opuntia explicaron $100 \%$ de la variación en sólo dos funciones discriminantes, excepto en las especies $O$. robusta y $O$. streptacantha, en las cuales se explicó 96 y 78.5\% de la variación explicada, respectivamente. El análisis discriminante detec- 
Cuadro 4. Análisis discriminante entre diferentes especies de Opuntia de la porción sur del Desierto Chihuahuense con base en variables morfológicas y anatómicas.

\begin{tabular}{lccc}
\hline & \multicolumn{3}{c}{ Función discriminante } \\
\cline { 2 - 4 } Variable & 1 & 2 & 3 \\
\hline Valor propio & 16.42 & 7.667 & 1.771 \\
Variación explicada (\%) & 59.8 & 6.4 \\
Variación explicada acumulada (\%) & 59.8 & 97.9 & 9.1 \\
Correlación canónica & 0.971 & Matriz de estructura \\
\hline Variable original & & 0.282 & 0.799 \\
\hline Profundidad de la cripta estomática & $0.493^{*}$ & 0.193 & -0.119 \\
Grosor de cutícula & $0.234^{*}$ & -0.029 \\
Altura del tallo & $0.196^{*}$ & 0.120 & 0.008 \\
Número de espinas adpresas por aréola & $0.175^{*}$ & -0.022 \\
Número de espinas menores de 1 cm por aréola & 0.163 & 0.077 & 0.220 \\
Grosor del parénquima & 0.032 & $0.256^{*}$ & 0.132 \\
Grosor del cladodio & 0.014 & $0.255^{*}$ & 0.165 \\
Grosor de la epidermis & 0.078 & $0.155^{*}$ & -0.121 \\
Número de líneas de aréolas por cara del cladodio & 0.416 & -0.412 & $0.488^{*}$ \\
Distancia máxima de la parte apical a la más ancha del cladodio & -0.011 & 0.241 & $0.437^{*}$ \\
Distancia entre líneas de aréolas & -0.212 & 0.379 & $0.423^{*}$ \\
Distancia máxima de la parte basal a la más ancha del cladodio & 0.040 & 0.259 & $0.268^{*}$ \\
Ancho de estomas & -0.061 & -0.103 & $0.216^{*}$ \\
Grosor del colénquima & -0.189 & 0.084 & $0.200^{*}$ \\
\hline
\end{tabular}

*correlaciones significativas $(\mathrm{P}<0.01)$ entre la variable original y la función discriminante.

tó nueve variables -el mayor número de variables originales que logró explicar $78 \%$ de la variación de $O$. streptacantha-, mientras que el análisis para $O$. leucotricha detectó seis variables, los realizados con $O$. rastrera y $O$. cantabrigiensis detectaron cinco variables, y en el de $O$. robusta sólo se detectaron tres variables originales, para explicar la variación total (cuadro 6).

Las variables originales que presentaron correlaciones significativas con las funciones discriminantes en más de una especie fueron el grosor de cladodio, la distancia máxima de la parte basal a la más ancha del cladodio, la distancia entre aréolas, el grosor del colénquima, el grosor de parénquima, la densidad estomática y el ancho de estomas (cuadro 6).

El diagrama de dispersión de $O$. cantabrigiensis muestra que las tres poblaciones estudiadas presentan marcadas diferencias a través de las dos primeras funciones discriminantes. Por influencia de la función 1 , las poblaciones de las localidades de El Rayo y Villa Hidalgo se encuentran ubicadas a la derecha de la figura $3 a$, ya que muestran valores mayores de distancia de la parte basal a la parte más ancha del cladodio, distancia entre aréolas y densidad estomática, que los individuos de la población de Laguna de Guadalupe. La función discriminante 2 separó las poblaciones de Villa Hidalgo y el Rayo, las cuales se distinguieron por tener diferencias en el tamaño de las aréolas y en el número de espinas porrectas por aréola (figura 3a).

En el diagrama de dispersión de $O$. leucotricha se observa que la población de Laguna de Guadalupe se ubicó en la parte superior derecha de la gráfica por la influencia de la función discriminante 1. Esta población se caracteriza por
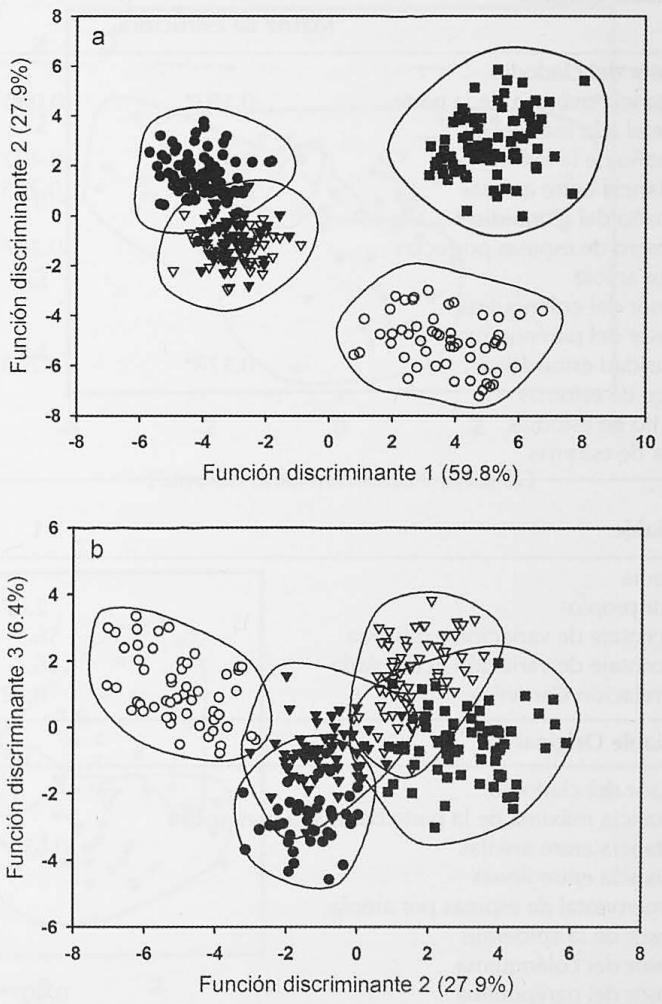

Figura 2. Diagrama de dispersión de las funciones discriminantes 1 y 2 (a) y diagrama de dispersión de las funciones discriminantes 2 y 3 (b) en Opuntia cantabrigiensis $(\Delta)$, O. leucotricha $(\bigcirc), O$. rastrera $(\boldsymbol{\nabla})$, O. robusta $(-$ y $O$. streptacantha $(\mathbf{i})$. 
Cuadro 5. Clasificación de los individuos de las diferentes especies de Opuntia a través del análisis discriminante.

\begin{tabular}{lcccccc}
\hline & & \multicolumn{5}{c}{ Pronóstico de membresía (\%) } \\
\cline { 3 - 7 } Especie & $N$ & OPCA & OPLE & OPRA & OPRO & OPST \\
\hline OPCA & 41 & 100 & 0 & 0 & 0 & 0 \\
OPLE & 54 & 0 & 100 & 0 & 0 & 0 \\
OPRA & 59 & 0 & 0 & 98.3 & 1.7 & 0 \\
OPRO & 80 & 1.3 & 0 & 0 & 98.8 & 0 \\
OPST & 84 & 0 & 0 & 0 & 0 & 100 \\
\hline
\end{tabular}

${ }^{*} \mathrm{OPCA}=\mathrm{O}$. cantabrigiensis, $\mathrm{OPLE}=\mathrm{O}$. leucotricha, OPRA $=0$. rastrera; $\mathrm{OPRO}=0$. robusta, $\mathrm{OPST}=O$. streptacantha. presentar menor grosor de los cladodios y del parénquima, así como mayor densidad estomática y mayor distancia entre aréolas, en comparación de la población de Villa Hidalgo, la cual quedó ubicada en la parte superior izquierda de la gráfica (figura 3b). En contraste, la población de El Rayo se encuentra distribuida ligeramente hacia la parte inferior de la gráfica por efecto de la función discriminante 2, la cual se correlacionó con el grosor del colénquima y el ancho de los estomas. Sin embargo, estas diferencias no son grandes, puesto que se observa yuxtaposición con el resto de las poblaciones (figura $3 b$ ).

Cuadro 6. Análisis discriminante entre diferentes poblaciones silvestres de Opuntia con base en variables morfológicas y anatómicas.

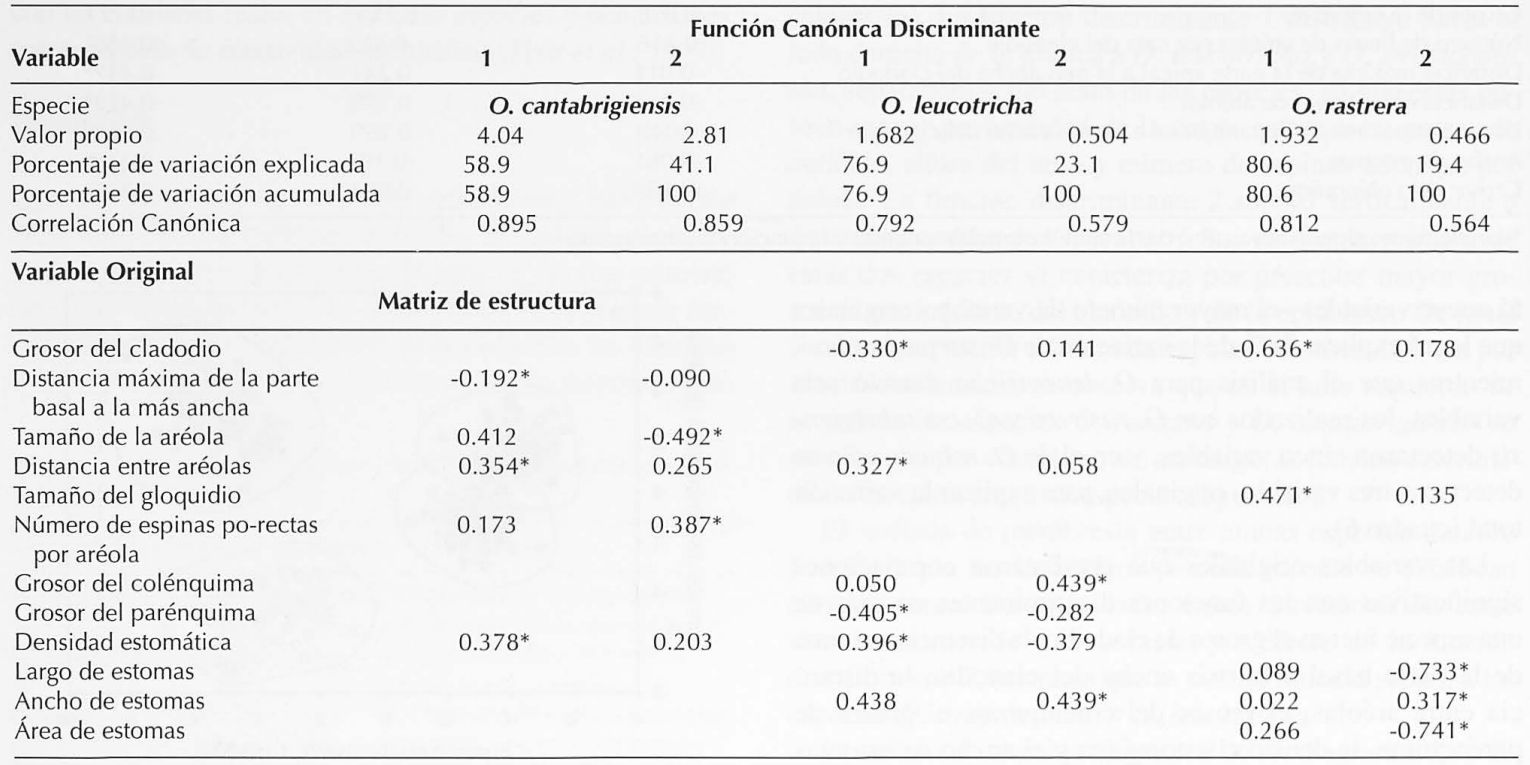

\begin{tabular}{|c|c|c|c|c|}
\hline \multirow[b]{2}{*}{ Variable } & \multicolumn{4}{|c|}{ Función Canónica Discriminante } \\
\hline & 1 & 2 & 1 & 2 \\
\hline Especie & & O. robusta & O. stre & \\
\hline Valor propio & 2.071 & 1.437 & 3.289 & 1.319 \\
\hline Porcentaje de variación explicada & 56.5 & 39.2 & 56 & 22.5 \\
\hline Porcentaje de variación acumulada & 56.5 & 95.7 & 56 & 78.5 \\
\hline Correlación Canónica & 0.821 & 0.768 & 0.876 & 0.754 \\
\hline Variable Original & & Matri & uctura & \\
\hline Grosor del cladodio & & & 0.159 & $0.282 *$ \\
\hline Distancia máxima de la parte basal a la mas ancha & & & $0.115^{*}$ & -0.020 \\
\hline Distancia entre aréolas & $-0.630^{*}$ & 0.295 & & \\
\hline Distancia entre líneas & & & 0.072 & $0.202 *$ \\
\hline Número total de espinas por aréola & & & 0.002 & $0.414^{*}$ \\
\hline Grosor de la epidermis & & & $0.537^{*}$ & -0.184 \\
\hline Grosor del colénquima & & & $-0.370^{*}$ & -0.016 \\
\hline Grosor del parénquima & $0.407^{*}$ & 0.227 & & \\
\hline Profundidad de la cripta estomática & & & -0.055 & $-0.129 *$ \\
\hline Densidad estomática & $0.529^{*}$ & 0.075 & $0.575^{*}$ & 0.464 \\
\hline Ancho de estomas & & & 0.140 & $-0.596 *$ \\
\hline
\end{tabular}

*correlaciones significativas $(P<0.01)$ entre la variable original y la función discriminante. 
La población de $O$. rastrera de El Rayo se encuentra ubicada en la parte inferior derecha de la gráfica (figura 3c), ya que presenta menor grosor de cladodio y sus gloquidios son de mayor tamaño, en comparación con las otras poblaciones. En contraste, la población de Villa Hidalgo muestra mayor grosor de cladodio y gloquidios más pequeños.
Por otro lado, los individuos de la población de Laguna de Guadalupe se yuxtaponen con los individuos de las otras poblaciones a pesar de tener estomas con mayor grosor pero menor área.

A pesar de que los individuos de $O$. robusta de las poblaciones de El Rayo y Laguna de Guadalupe se distinguieron

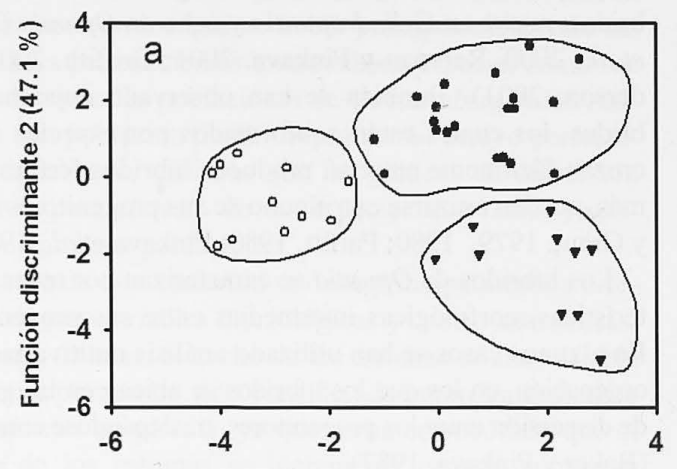

Función discriminante $1(52.9 \%)$

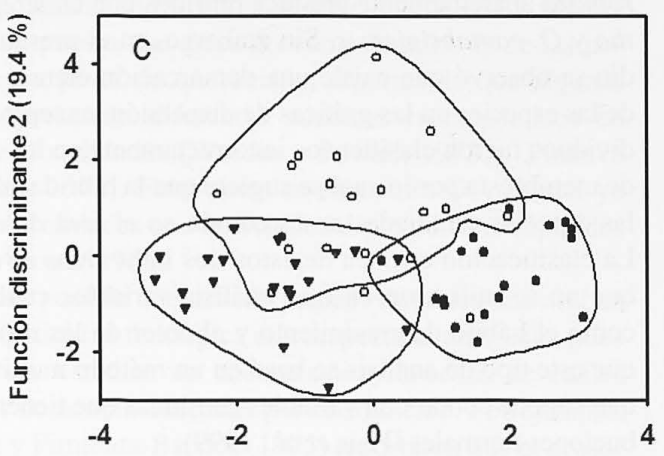

Función discriminante $1(80.6 \%)$

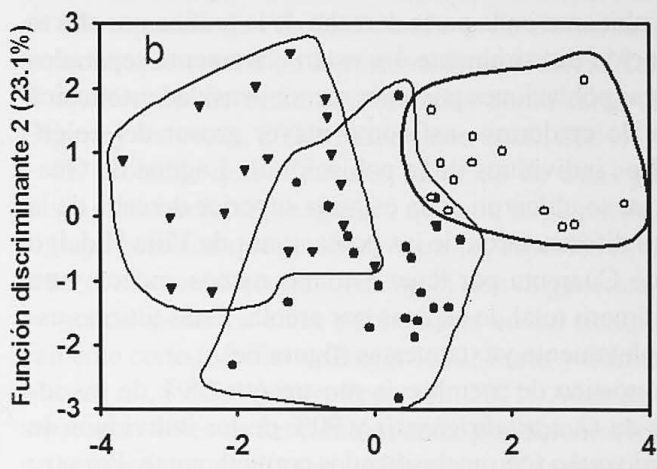

Función discriminante $1(76.9 \%)$

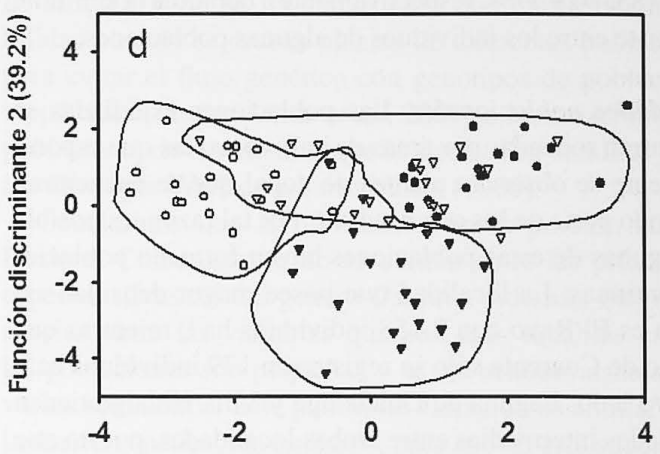

Función discriminante 1 (56.5\%)

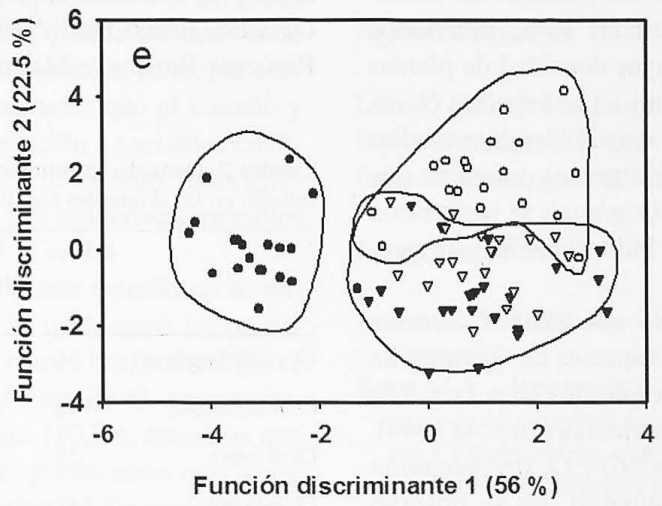

Figura 3. Diagrama de dispersión de las funciones discriminantes 1 y 2 en las poblaciones de Paso de Cuarenta $(\Delta)$, Villa Hidalgo $(\nabla)$, Laguna de Guadalupe (O) y El Rayo (○) de O. cantabrigiensis (a), O. leucotricha (b), O. rastrera (c), O. robusta (d) y O. streptacantha (e) 
por tener diferencias en la distancia entre líneas de aréolas, la densidad de estomas y el parénquima, los individuos de las poblaciones de Paso de Cuarenta se yuxtaponen con los individuos de El Rayo y Laguna de Guadalupe, mientras que los individuos de Villa Hidalgo se yuxtaponen ligeramente con individuos de las tres poblaciones (figura $3 \mathrm{~d}$ ).

En $O$. streptacantha los individuos de la población de El Rayo se ubicaron en la parte derecha de la gráfica por efecto de la función discriminante 1 y están claramente separados de las otras poblaciones por tener menor densidad estomática y grosor de epidermis, así como mayor grosor del colénquima. Los individuos de la población de Laguna de Guadalupe que se ubicaron en la esquina superior derecha de la gráfica se diferenciaron de las poblaciones de Villa Hidalgo y Paso de Cuarenta por tener estomas menos anchos y un menor número total de espinas por aréola. Estas últimas están completamente yuxtapuestas (figura 3e).

El pronóstico de membresía mostró que $98 \%$ de los individuos de $O$. cantabrigiensis y $90 \%$ de los individuos de $O$. streptacantha fueron clasificados correctamente. Por otro lado, las especies $O$. leucotricha, $O$. rastrera y $O$. robusta exhiben menor porcentaje de individuos clasificados correctamente $(85,80$ y $77 \%$, respectivamente); debido a la similitud que existe entre los individuos de algunas poblaciones.

Densidades poblacionales. Las poblaciones estudiadas se encuentran rodeadas por áreas de cultivo, en las que esporádicamente se observan plantas de nopal que se encuentran formando parte de los cercos vivos. Por tal razón, es posible que algunas de estas poblaciones hayan formado poblaciones continuas. La localidad que posee mayor densidad estimada es El Rayo con 2,458 individuos ha-1, mientras que en Paso de Cuarenta sólo se registraron 179 individuos ha-1. Por otro lado, Laguna de Guadalupe y Villa Hidalgo tienen densidades intermedias entre ambas localidades, puesto que existen 710 y 680 individuos ha-1 ${ }^{-1}$, respectivamente.

La especie con la mayor densidad fue $O$. streptacantha con un promedio de 596 individuos ha-1, aunque en localidades como El Rayo fueron registradas 1,852 individuos $\mathrm{ha}^{-1}$, y ésta fue la población con mayor densidad de plantas observada entre las especies en estudio. Las especies $O$. robusta y $O$. leucotricha mostraron densidades intermedias (138 y 112 individuos ha $^{-1}$ respectivamente), mientras que las especies $O$. rastrera y $O$. cantabrigiensis se registraron las densidades más bajas ( 71 y 23 individuos ha-1, respectivamente; cuadro 7).

Números cromosómicos. Se observó que existen diversos niveles de ploidía entre las diversas especies de Opuntia. Se encontró que $O$. cantabrigiensis es diploide $(2 \mathrm{n}=2 \mathrm{x}=22)$; $O$. leucotricha y $O$. robusta son tetraploides $(2 n=4 x=44)$, $O$. rastrera es hexaploide $(2 \mathrm{n}=6 \mathrm{x}=66)$ y $O$. streptacantha octoploide $(2 \mathrm{n}=8 \mathrm{x}=88)$. Sin embargo, no se observó variación en el número cromosómico entre poblaciones, lo que nos permite sugerir que la variación morfológica y anatómica que se observa en algunas especies no se relaciona con los niveles de ploidía observados.

\section{Discusión}

La hibridación es un fenómeno común en los géneros Opuntia y Cylindropuntia, ya que se han registrado más de 17 híbridos; nueve en Cylindropuntia y ocho en Opuntia (Mayer et al., 2000, Rebman y Pinkava, 2001; Griffith, 2001; Anderson, 2001). También se han observado enjambres híbridos, los cuales están conformados por especies que se cruzan fácilmente entre sí, producen híbridos fértiles y aun más, pueden cruzarse con alguno de sus progenitores (Grant y Grant, 1979, 1980; Parfitt, 1980; Pinkava et al., 1985).

Los híbridos de Opuntia se caracterizan por tener características morfológicas intermedias entre sus progenitores. En algunos casos se han utilizado análisis multivariados de ordenación, en los que los híbridos se ubican en las gráficas de dispersión entre los progenitores, traslapándose entre ellos (Baker y Pinkava, 1987).

Respecto a la hibridación entre las especies correspondientes a este estudio Bravo-Hollis (1978) menciona que $O$. robusta aparentemente produce híbridos con $O$. streptacantha y $O$. cantabrigiensis. Sin embargo, en el presente estudio se observó que existe una demarcación clara y precisa de las especies en las gráficas de dispersión, excepto dos individuos fueron clasificados incorrectamente en los análisis de membresía por lo cual se sugiere que la hibridación entre las especies estudiadas no es común en el área de estudio. La clasificación errónea de estos dos individuos se debió a que no se utilizaron en este análisis variables cualitativas como el hábito de crecimiento y el color de las espinas ya que este tipo de análisis se basó en un método multivariado que supone contar con variables continuas que tienen distribuciones normales (Hair et al., 1999).

$\mathrm{El}$ hecho de que los individuos hayan estado perfectamente delimitados por el análisis discriminante no descarta la amplia variación morfológica que ha sido reportada en Opuntia (Bravo-Hollis, 1978; Wallace y Fairbrothers, 1986; Pimienta-Barrios y Mauricio-Leguizamo, 1989; Pimienta-

Cuadro 7. Densidades estimadas (individuos ha-1) de las especies de estudio en las diferentes localidades muestreadas.

\begin{tabular}{lrrrrr}
\hline & El Rayo & $\begin{array}{c}\text { Villa } \\
\text { Hidalgo }\end{array}$ & $\begin{array}{c}\text { Laguna de } \\
\text { Guadalupe }\end{array}$ & $\begin{array}{r}\text { Paso de } \\
\text { Cuarenta }\end{array}$ & $\begin{array}{r}\text { Densidad } \\
\text { Promedio } \\
\text { por especie }\end{array}$ \\
\hline O. cantabrigiensis & 56 & 13 & 25 & 0 & 24 \\
O. leucotricha & 142 & 287 & 19 & 0 & 112 \\
O. rastrera & 25 & 87 & 173 & 0 & 71 \\
O. robusta & 142 & 106 & 241 & 62 & 138 \\
O. streptacantha & 1852 & 198 & 216 & 117 & 596 \\
\hline
\end{tabular}


Barrios, 1994; Pimienta-Barrios y Muñoz-Urias, 1995; Scheinvar, 1995). Sin embargo, en esta investigación se encontró variación entre las poblaciones estudiadas, habiéndose encontrando algunas diferencias en poblaciones aisladas de forma similar a los resultados obtenidos por Bruschi et al. (2003) en su estudio con Quercus petrea.

La variación entre las poblaciones fue observada en aquellos casos en los que no había yuxtaposición entre las poblaciones pertenecientes a una misma especie, como fue el caso de todas las poblaciones de $O$. cantabrigiensis. Asimismo, una población de $O$. streptacantha estuvo perfectamente delimitada, mientras que las gráficas de dispersión mostraron para el resto de las especies que había yuxtaposición entre sus poblaciones.

La causa de esta variación se debe principalmente a diferencias en tres variables morfológicas y cuatro anatómicas. Entre estas últimas, el ancho de los estomas es una variable importante porque ha sido considerada como un indicador de los niveles de ploidía, ya que se ha corroborado que el tamaño de los estomas se incrementa cuando aumentan los niveles de ploidía en Opuntia y en diversas especies (Conde, 1975). Sin embargo, no podemos relacionar la diferencia de tamaño en los estomas con cambios en los niveles de ploidía, puesto que estos no variaron a través de las poblaciones, a pesar de que existen reportes de que algunas especies presentan diferentes números cromosómicos en diferentes localidades (Weedin y Powell, 1978 y Pinkava y McLeod, 1971). Entre éstas se encuentra O. robusta, ya que hay localidades con individuos diploides o tetraploides (Sosa y Acosta, 1966).

No fue posible identificar cuáles variables mostraban menor plasticidad fenotípica con respecto al ambiente, pues en diversos estudios no encontramos consenso de cuáles variables no son modificadas por el ambiente. Por ejemplo, Ramírez y Pimienta-Barrios (1995) reportaron que el grosor del cladodio, el ancho de estomas, la densidad estomática y el grosor del colénquima son variables estables, mientras que Silva et al. (2001) reportan que el tamaño y la densidad de los estomas en plantas del género Opuntia pueden variar por diferencias en la exposición a la radiación solar, por otro lado Hernández et al. (2007) encontraron que el tamaño y la densidad de estomas varía con relación a variables edáficas en Myrtillocactus geometrizans. Por lo tanto, estas diferencias podrían estar influenciadas por numerosas variables ambientales.

Es importante hacer notar que en este estudio no se encontró una relación entre la distancia que separa las poblaciones y el grado de diferenciación entre ellas, puesto que se observó yuxtaposición entre poblaciones de $O$. streptacantha separadas por distancias de hasta $110 \mathrm{~km}$, mientras que no observamos yuxtaposición en dos poblaciones que se encuentran separadas por $32 \mathrm{~km}$ de distancia. De manera similar, se observa que en poblaciones de $O$. leucotricha separadas por $58 \mathrm{~km}$ hay yuxtaposición entre ambas poblaciones, pero que no existe yuxtaposición en poblaciones separadas por $32 \mathrm{~km}$.

La variación entre poblaciones fue mayor en $O$. cantabrigiensis, ya que no encontró yuxtaposición para ninguna de sus poblaciones. Esto se puede atribuir al hecho de que la densidad de las poblaciones en esta especie es la más baja, además sus abundancias son comparables con las densidades de las especies de Neobuxbaumia o de Polaskia chende, especies que crecen en la reserva de la biósfera TehuacánCuicatlán, donde están consideradas como raras (Esparza Olguín y Valverde, 2003; Mandujano et al., 2004).

Es probable que el tamaño reducido de estas poblaciones y el aislamiento geográfico que a la larga puede reducir el flujo genético que se observa en las especies estudiadas, sean condiciones que favorezcan la deriva genética y la divergencia genética entre poblaciones en un tiempo relativamente corto (Mettler y Gregg, 1972; Jonas y Gerber, 1999; Max et. al., 1999; Walter y Epperson, 2001). Además, pueden ser responsables de los cambios entre poblaciones, los cuales no responden a gradientes ambientales (Karakousis y Skibinski, 1992; Wen y Hsiao, 1999; Renaud y Millien, 2001).

Hay otras evidencias de divergencia genética, ya que en $O$. streptacantha se ha demostrado que existe incompatibilidad cuando se cruzan plantas de diferentes poblaciones para evitar el fiujo genético con genotipos de poblaciones alopátricas (Muñoz-Urias et al., 2006) o la variación interpoblacional de isoenzimas que se detectó en semillas de $O$. humifusa por Wallace y Fairbrothers (1986).

Concluimos que la hibridación en la porción sur del Desierto Chihuahuense no es común entre las principales especies silvestres del género Opuntia. Además, hubo diferencias entre las variables que son más notables cuando las poblaciones son pequeñas y se encuentran aisladas por varios kilómetros. Este patrón de variación es similar al de la deriva genética; sin embargo, para apoyar esta sugerencia sería conveniente realizar estudios sobre genética molecular en estas poblaciones para confirmar esta hipótesis.

\section{Agradecimientos}

Lucero Quiroz Rocha proporcionó valiosa ayuda en el trabajo de campo. La Universidad de Guadalajara otorgó el financiamiento para la realización de este proyecto.

\section{Literatura citada}

Anderson E.F. 2001. The Cactus Family. Timber Press, Portland, Oregon.

Baker M.A. y Pinkava D.J. 1987. A cytological and morphometric analysis of a triploid apomictic, Opuntia $\times$ kelvinensis (subgenus Cylindropuntia, Cactaceae). Brittonia 39:387-401.

Bravo-Hollis H. 1978. Las Cactáceas de México, Vol. 1. Universidad Nacional Autónoma de México, México, D.F.

Britton N.L. y Rose J.N. 1963. The Cactaceae. Descriptions and Illustrations of Plants of the Cactus Family. Vol. 1. Courier Do- 
ver Publications, Nueva York.

Brower J.B., Zar J.H. y von Ende C. N. 1997. Field and Laboratory Methods for General Ecology. Mc Graw-Hill, Dubuque.

Bruschi P., Vendramin G.G., Bussotti F. y Grossoni P. 2003. Morphological and molecular diversity among Italian populations of Quercus petraea (Fagaceae) Annals of Botany 91:707-716.

Conde L.F. 1975. Anatomical comparisons of five species of Opuntia (Cactaceae). Annals of the Missouri Botanical Garden 62:425-473

Ellstrand N.C. y Elam R.D. 1993. Population genetic consequences of small population size: implications for plant conservation. Annual Review of Ecology and Systematics 24: 217-242.

Esparza Olguín L. y Valverde T. 2003. Estudio comparativo de la fenología de tres especies de Neobuxbaumia que difieren en su nivel de rareza. Cactáceas y Suculentas Mexicanas 48:68-83.

García Velázquez, A. 1988. Técnicas y Procedimientos de Citogenética Vegetal. Colegio de Postgraduados. Montecillo, Estado de México.

Gastó C.J., Nava C.R. y López J.J. 1981. Proceso de carga y descarga frutal en poblaciones naturales de Opuntia streptacantha Lemaire. Monografía Técnico-Científica. Vol. 7 Universidad Autónoma Agraria Antonio Narro. Pp. 170-216.

Gibson A. y Nobel P.S. 1986. The Cactus Primer. Harvard University Press. Cambridge, MA.

Grant V. y Grant K.A. 1979. Hybridization and variation in the Opuntia phaeacantha group in central Texas. Botanical Gazette 140:208-215.

Grant V. y Grant K.A. 1980. Clonal microspecies of hybrid origin of Opuntia lindheimeri group. Botanical Gazette 141:101-106.

Griffith P.M. 2001. A new Chihuahuan Desert prickly pear, Opuntia $\times$ rooneyi (Cactaceae). Cactus and Succulent Journal (U.S.) 73:307-310.

Hair J.F., Anderson R.E., Tatham R.L. y Black W.C. 1999. Análisis Multivariante. Prentice Hall. Madrid.

Hernández M., Terrazas T., Delgado-Alvarado A. y Luna-Cavazos M. 2007. Los estomas de Myrtillocactus geometrizans (Mart. Ex. Pfeiff.) console (Cactaceae): variación en su área de distribución. Revista Fitotecnia Mexicana 30:235-240.

Janzen D.H. 1986. Chihuahuan Desert nopaleras: defaunated big mammal vegetation. Annual Review of Ecology and Systematics 17:595-636.

Jonas C.S. y Gerber M.A. 1999. Variation among populations of Clarkia unguiculata (Onagraceae) along altitudinal and latitudinal gradients. American Journal of Botany 86:333-343.

Karakousis Y. y Skibinski D.O.F. 1992. An analysis of allozyme, mitochondrial DNA and morphological variation in mussel (Mytilus galloprovincialis) populations from Greece. Cellular and Molecular Life Science 48:878-881.

Mandujano M.C., Golubov J., Rodríguez-Ortega C., Reyes J., Aguilar G. y Rojas-Aréchiga M. 2004. Estructura poblacional y distribución de poblaciones de Polaskia chende (Gosselin) Backeb., en un municipio dentro de la reserva de la biosfera de Tehuacán-Cuicatlán. Cactáceas y Suculentas Mexicanas 49:3661.

Marroquín S.G., Borja L., Velázquez C.R. y De la Cruz J.A. 1964. Estudio Ecológico Dasonómico de las Zonas Áridas del Norte de México. Publicación especial Número 2 Instituto Nacional de Investigaciones Forestales, Secretaría de Agricultura y Recursos Hidráulicos, México, D.F.

Max K.N., Mouchaty S.K. y Schwaegerle K.E. 1999. Allozyme and morphological variation in two subspecies of Dryas octopetala (Rosaceae) in Alaska. American Journal of Botany 86:1637-1644.

Mayer M.S., Williams L.M. y Rebman J.P. 2000. Molecular evidence for the hybrid origin of Opuntia prolifera (Cactaceae). Madroño 47:109-115.

Mc Leod M.G. 1975. A new hybrid fleshy-fruited prickly-pear in California. Madroño 23:96-98.

Mettler L.E. y Gregg T.G. 1972. Genética de Poblaciones y Evolución. UTEHA. México, D.F.

Muñoz-Urias A., García-Velázquez A. y Pimienta-Barrios E. 1995. Relación entre el nivel de ploidía y variables anatómicas y morfológicas en especies silvestres y cultivadas de nopal tunero (Opuntia spp.). En: Pimienta-Barrios E., Neri-Luna C., Muñoz-Urias A. y Huerta-Martínez F. M. Comps. Conocimiento y Aprovechamiento del Nopal, 6to Congreso Nacional y 4to Congreso Internacional, pp. 7-11, Universidad de Guadalajara, Guadalajara.

Muñoz-Urias A., Palomino-Hasbach G., Huerta-Martínez F.M., Pimienta Barrios E. y Ramírez-Hernánez B.C. 2006. Reproductive isolation in fragmented wild populations of Opuntia streptacantha. Journal of the Professional Association for Cactus Development 8:26-38.

Orea-Rosas, T. B. 1986. Variación morfológica de Opuntia spp en nopales silvestres del suroeste del desierto Chihuahuense. Tesis Licenciatura (Ingeniero Agrónomo en Fitotecnia). Universidad Autónoma de San Luís Potosí. 90 pp.

Palomino G. y Heras H.M. 2001. Karyotypic studies in Opuntia cochinera, O. hyptiacantha, and $O$. streptacantha (Cactaceae). Caryologia 54:147-154.

Parffit B.D. 1980. Origin of Opuntia curvospina (Cactaceae). Systematic Botany 5: 408-418.

Pimienta-Barrios E. 1990. El Nopal Tunero. Serie libros tiempos de ciencia, Universidad de Guadalajara. Guadalajara.

Pimienta-Barrios E. 1994. Prickly pear (Opuntia spp.): a valuable fruit crop for the semi-arid lands of Mexico. Journal of Arid Environments 28:1-11

Pimienta-Barrios E. y Mauricio-Leguizamo R. 1989. Variación en componentes del fruto maduro entre formas de nopal (Opuntia spp.) tunero. Revista Fitotecnia Mexicana 12:183-196.

Pimienta-Barrios E. y Muñoz-Urias A. 1995. Domestication of opuntias and cultivated varieties. En: Barbera G., Inglese P., Pimienta-Barrios E. Eds. Agro-ecology, Cultivation and Uses of Cactus Pear, pp. 58-61, FAO, Roma.

Pinkava D.J. y Mc Leod M.G. 1971. Chromosome numbers in some cacti of western North America. Brittonia 23:171-176.

Pinkava D.J., Baker M.A., Parffit B.D. y Mohlenbrock M.W. 1985. Chromosome numbers in some cacti of western North AmericaV. Systematic Botany 10:471-483.

Pinkava D.J., Mc Gill L.A., Reeves T. y Mc Leod M.G. 1977. Chromosome numbers in some cacti of western North America -III. Bulletin of Torrey Botanical Club 104:105-110.

Pinkava D.J., Mc Leod M.G., Mc Gill L.A. y Brown R.C. 1973. Chromosome numbers in some cacti of western North America - II. Brittonia 25:2-9.

Pinkava D.J., Parffit B.D., Baker M.A. y Worthington R.D. 1992. Chromosome numbers in some cacti of western North America - VI, with nomenclatural changes. Madroño 39:98-113.

Ramírez A.J.P. y Pimienta-Barrios E. 1995. El efecto del grado de aridez en la anatomía de cladodios de morfoespecies de nopal 
(Opuntia spp). En: Pimienta-Barrios E., Neri-Luna C., MuñozUrias A. y Huerta-Martínez F.M. (Eds.). Conocimiento y Aprovechamiento del Nopal. Memorias del 6to Congreso Nacional y 4to Congreso Internacional, pp. 90-99, Universidad de Guadalajara, Guadalajara.

Rebman J.P. y Pinkava D.J. 2001. Opuntia cacti of North Americaan overview. Florida Entomologist 84:474-483.

Renaud S. y Millien V. 2001. Intra- and interspecific morphological variation in the field mouse species Apodemus argenteus and A. speciosus in the Japanese archipelago: the role of insular isolation and biogeographic gradients. Biological Journal of the Linnean Society 74:557-569.

Scheinvar L. 1995. Taxonomy of utilized opuntias. En: Barbera G., Inglese P., Pimienta-Barrios E. Eds. Agro-Ecology, Cultivation and Uses of Cactus Pear, pp. 20-27, FAO, Roma.

Silva H., Acevedo E. y Silva P. 2001. Anatomía del tejido fotosintético de diez taxa de Opuntia establecidos en el secano árido mediterráneo de Chile. Revista Chilena de Historia Natural 74:341-351.

Sosa R. y Acosta A. 1966. Poliploidia en Opuntia spp. Agrociencia 1:100-106.

Starmer W.T., Schmedicke R.A. y Lachance M. 2003. The origin of the cactus-yeast community. Federation of European Microbiological Society Yeast Research 3:441-448.

Valverde P.L., Vite F. y Zavala-Hurtado J.A. 1996. A morphometric analysis of a putative hybrid between Agave marmorata Roezl and Agave kerchovei Lem.: Agave peacockii Croucher. Botani- cal Journal of the Linnean Society 122:155-161.

Vite F., Portilla E., Zavala-Hurtado J.E., Valverde, P.L. y Díaz-Solís A. 1996. A natural hybrid population between Neobuxbaumia tetetzo and Cephalocereus columna-trajani (Cactaceae). Journal of Arid Environments 32:395-405.

Walter R. y Epperson B.K. 2001. Geographic pattern of genetic variation in Pinus resinosa: area of greatest diversity is not the origin of postglacial populations. Molecular Ecology 10:103 111 .

Wallace R.S. y Fairbrothers D. 1986. Isoelectrically focussed seed proteins of populations of Opuntia humifusa (Raf.) Raf. (Cactaceae). Biochemical Systematics and Ecology 14:365-369.

Weedin J.F. y Powell A.M. 1978. Chromosome numbers in Chihuahuan Desert cactaceae. Trans-Pecos, Texas. American Journal of Botany 65:531-537.

Wen C.-S. y Hsiao J.-Y. 1999. Genetic differentiation of Lilium longiflorum Thunb. var. scabrum Masam. (Liliaceae) in Taiwan using Random Amplified Polymorphic DNA and morphological characters. Botanical Bulletin of Academia Sinica 40:65-71.

Young A.G. y Clarke G.M. 2000. Genetics, Demography and Viability of Fragmented Populations. Cambridge University Press, Cambridge.

Zar J.H. 1984. Biostatistical Analysis. Prentice-Hall. Upper Saddle River, Nueva Jersey.

Zelaya I.A., Owen M.D.K. y Vangessel M.J. 2007 Transfer of glyphosate resistance: evidence of hybridization in Conyza (Asteraceae). American Journal of Botany 94:660-673. 